\title{
Molecular modelling of the interaction of carbocyclic analogues of netropsin and distamycin with d(CGCGAATTCGCG)2
}

\author{
Krzysztof Bielawski ${ }^{1 凶}$, Anna Bielawska ${ }^{1}$, Danuta Bartulewicz ${ }^{2}$ and Andrzej Różański ${ }^{2}$ \\ ${ }^{1}$ Department of Medicinal Chemistry and Drug Technology, Medical Academy of Biatystok, \\ Biatystok, Poland; ${ }^{2}$ Department of Organic Chemistry, Medical Academy of Biatystok, Poland
}

Received: 24 January, 2000; accepted: 05 June, 2000

Key words: DNA-ligand interaction, carbocyclic analogues of netropsin and distamycin, molecular modelling, netropsin, distamycin

\begin{abstract}
A molecular mechanics and molecular dynamics approach was used to examine the structure of complexes formed between the d(CGCGAATTCGCG) 2 duplex and netropsin, distamycin, and four carbocyclic analogues of netropsin and distamycin (1-4). The resulting structures of the ligand-DNA model complexes and their energetics were examined. It is predicted that the compounds 1-4 should have a decreased affinity for the minor groove of AT-rich regions in comparison to netropsin and distamycin. From the energetic analysis it appears that van der Waals and electrostatic interactions are more important than specific hydrogen bonds in stabilizing the ligand-duplex complexes. We predict that compounds 1 and 2 are effectively isohelical with the DNA minor groove. The superior DNA-binding afforded by 1 and 2 in comparison to 3 and 4 results from their more effective penetration into the minor groove and smaller perturbation of molecular structure upon complex formation.
\end{abstract}

Nucleic acids are important targets in the chemotherapeutic treatment of diseases and neoplasms. Interference with gene function and prevention of transcription and translation can kill invading microorganisms or tumour cells. A number of natural and synthetic compounds are known to bind to DNA double helix in a nonintercalative manner [1, 2]. This is possible because most DNA-binding molecules possess cationic functional groups, complementary in size to one of the grooves, have an aromatic ring system, or a combination factors of these [1]. The structure of two well-known compounds of this type, the antibiotics netropsin and distamycin is shown in Fig. 1. Biophysical and footprinting studies

\footnotetext{
${ }^{\otimes}$ Author for correspondence: Krzysztof Bielawski, Department of Medicinal Chemistry and Drug Technology, Medical Academy of Białystok, 15-230 Białystok 8, J. Kilińskiego 1, Poland; phone: (48 85) 742 4710; fax: (48 85) 742 4907; e-mail: kbiel@amb.ac.bialystok.pl
} 
have shown that these compounds bind in the narrow minor groove of B-DNA by means of hydrogen bonds, ionic charge attractions, as well as van der Waals interactions [3-5]. They have a binding preference to stretches of AT-rich regions over GC base pairs because binding to the latter is sterically hindered by
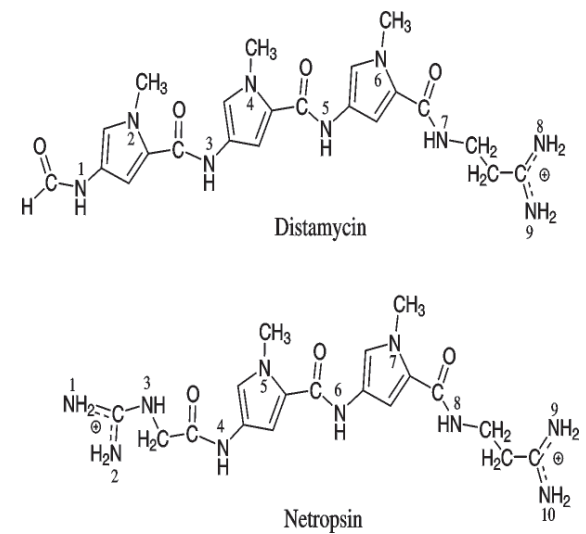

Figure 1. The structures of netropsin and distamycin, with nitrogen atoms numbered.

the presence of the bulky 2-amino group of the guanine residue that protrudes into the minor groove [3]. Further, the deepest negative potential wells in the minor groove of DNA occur at $(\mathrm{AT})_{\mathrm{n}}$ sequences ensuring that biscationic netropsin analogues gravitate to such sequences [6]. Such sequence binding microheterogeneity is now better understood based on the recent structural information available from several X-ray diffraction analyses of drug-DNA dodecamer complexes [3, 4].

The synthesis and testing of analogues of netropsin and distamycin is a subject of active research [2, 7-9]. These studies clearly show that the length of three pyrrole rings is optimal for binding of this class of compounds, and a further increase in the number of heterocycles to target for a longer DNA sequence is not feasible due to the phasing incompatibility [8] which arises because of the lack of dimensional correspondence between oligopeptides and oligonucleotides. It has been found that a sequence-specific ligand binding to a longer specificity region of DNA is best obtained by connecting two netropsins or their analogues together via a short con- necting chemical group or linker [8, 9]. Alternatively, we can develop the strategy that consists in replacing the N-methylpyrrole rings with new subunits of an appropriate repeat length that permits to retain the attractive features of netropsin and distamycin [8, 10]. We focused on the strategy that consists in replacing the N-methylpyrrole rings with carbocyclic rings with a minor modification of cationic heads (Fig. 2). Carbocyclic analogues of netropsin and distamycin are readily available, can be modified easily, and are stable under most experimental conditions [10-12]. Recently, compounds 1-4 which were investigated on the standard cell line of mammalian tumour MCF-7, revealed high antitumour activity [12]. In order to improve their sequence selectivity and overall DNA-binding properties, it is important to understand in detail their mode of interaction at the molecular

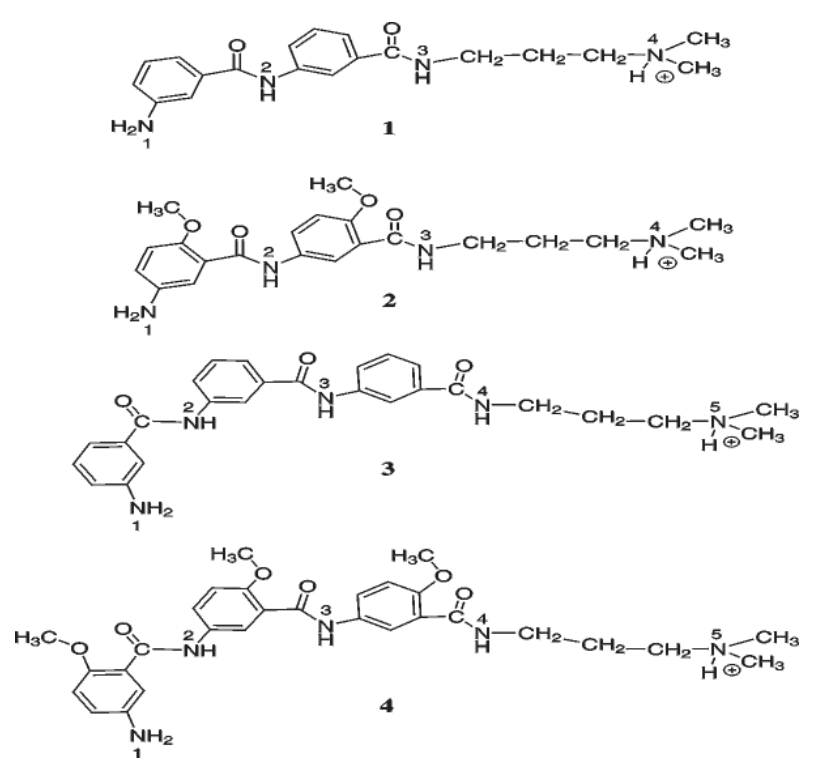

Figure 2. The structures of carbocyclic analogues 1-4 of netropsin and distamycin, with nitrogen atoms numbered.

level. Consequently, computational methods can be useful in modelling ligand-DNA asso- 
ciations to predict the structure and probe the stereochemistry of recognition. We have examined a representative range of carbocyclic analogues of netropsin and distamycin (compounds 1-4 in Fig. 2) in terms of a computed binding energy with a model DNA sequence and structure-activity relationships using combined molecular mechanics and molecular dynamics methods.

\section{METHODS}

Chemistry. The synthesis of compounds 1-4, together with complete spectral characterization, has been reported in the previous paper [12].

Molecular modelling. Initial structures for netropsin, distamycin and molecules 1-4 (Fig. 1 and 2) were constructed using the HyperChem version 5.11 program, which was also used for manipulation and interactive docking manoeuvres. This program was run on an Intel Pentium II $450 \mathrm{MHz}$ personal computer.

Initial coordinates for the 12-mer DNA duplex host were taken from the crystal structure of the d(CGCGAATTCGCG) $)_{2}$ complex from the Brookhaven Protein Databank (PDB file 1bna) [13]. Initial models for each complex were constructed by docking the ligands at a core A4-T7 location within the minor groove with a) 1:1 stoichiometry, b) the concave surface of the molecules facing the convex groove floor, and c) fully extended, alltrans ligand conformations. Ligands 1-4 are asymmetric in structure, therefore two alternative orientations of these for compounds in the minor groove of DNA were considered. A rigid-body refinement procedure was used to align the ligand molecules with the walls of the minor groove, and to remove unfavourable atomic contacts, prior to energy minimisation. For netropsin, distamycin and 1-4, alternative positions of the compounds were considered by translation of the ligand along the 5'-AATT-3' minor groove tract of the
DNA. Ligand displacements were restricted \pm 2 bp from an initial location. The AMBER force field [14-16] (as implemented in HyperChem 5.11) was used for all energy calculations. Additional non-standard AMBER force-field parameters required for the ligands were derived here by interpolation. Atom-centered charges for each molecule were computed from the AM1 wavefunctions (HyperChem 5.11) by the procedure of Orozco \& Luque [17], which provides derived charges that closely resemble those obtainable from $a b$ initio $6-31 \mathrm{G}^{*}$ calculations.

In the case of compounds 1-4, the molecular modelling is further complicated by different distinct conformations available for the ligand molecules. These compounds can adopt structures with the carboxamide groups in various potential orientations with respect to one another. The structural demands of the receptor may lead to binding of the molecule in a conformation that would not be favoured in its nonbound state. Models were constructed for possible ligand conformations differing in the relative orientation of the carboxamide group and terminal amino group with respect to the minor groove (Fig. 3A and $3 \mathbf{B})$ and were subjected to molecular mechanics energy minimization to establish the effect of conformation upon DNA binding. Ranking orders of $\mathbf{1} \mathrm{A}>\mathrm{B}>\mathrm{C}>\mathrm{D} ; \mathbf{2} \mathrm{A}>\mathrm{B}>\mathrm{D}$; $3 \mathrm{~A}>\mathrm{B}>$ $\mathrm{D}>\mathrm{C}>\mathrm{E} ; \mathbf{4} \mathrm{A}>\mathrm{B}>\mathrm{C}>\mathrm{E}$ were determined for the ligands. On this basis, models 1A, 2A, 3A and $4 \mathrm{~A}$ were selected for subsequent molecular dynamics refinement (see below).

The DNA-ligand complexes were initially regularized by conjugate-gradient molecular modelling to reduce poor intermolecular steric contacts so as to minimize the energy of the bound ligand alone and for minimization of the unrestrained complex to an energy gradient of $<0.01 \mathrm{kcal} /(\AA \times \mathrm{mol})$. Molecular dynamics (MD) simulations of each complex were subsequently performed for 5 ps (integration time step $=1 \mathrm{fs}$ ) at $300 \mathrm{~K}$. Potential energy analysis during molecular dynamics progress showed that the systems reached 
equilibrium rapidly, typically at [times of] $<2$ ps. Atomic coordinates were sampled at 0.2-ps intervals during the simulation period. In each case, the averaged structure from the accumulated snapshots was subjected to final molecular mechanics relaxation. The Polak-Ribiere minimization method was applied with a gradient value of 0.01 to test for convergence, to generate the refined complex. van der Waals and H-bonded energy terms were included up to $8 \AA$, but explicit base pair restraints were not used. Solvent and counterions were not included explicity for reasons of computational expense. Instead, their effect was simulated using a simple distance-dependent dielectric constant with $\varepsilon=$ $4 \mathrm{r}_{\mathrm{ij}}$. This formalism is well established in the field of protein modelling and has been tested in some detail for a nucleic acid model system with satisfactory results $[14,18]$. Distance restraints corresponding to Watson-Crick base-paired geometry were not included, and no attempt was made to restrain either terminal base pairs or the DNA backbone. Binding energy and component perturbation energies were computed using:

$$
\begin{gathered}
\Delta E_{\text {bind }}=\Delta E_{\text {per }}(\text { ligand })+\Delta E_{\text {per }}(\text { DNA })+ \\
\Delta E_{\text {inter }} \text { and } \Delta E_{\text {inter }}=\Delta E_{\mathrm{vdw}}+\Delta E_{\mathrm{q}}+ \\
\Delta E_{\mathrm{HB}}
\end{gathered}
$$

where $\Delta E_{\text {bind }}$ is the energy of binding, $\Delta E_{\text {inter }}$ is the ligand-DNA interaction energy, and the $\Delta E_{\text {per }}$ terms represent the component perturbation energies for the ligand and DNA, relative to the unbound species. The decomposed van der Waals $\left(\Delta E_{\mathrm{vdw}}\right)$, electrostatic $\left(\Delta E_{\mathrm{q}}\right)$ and H-bonded $\left(\Delta E_{\mathrm{HB}}\right)$ binding terms are given by

$$
\begin{gathered}
\Delta E_{\text {term }}=E_{\text {term (complex) }}-\left(E_{\text {term }(\mathrm{DNA})}+\right. \\
\left.E_{\text {term (ligand free })}\right)
\end{gathered}
$$

Perturbation energies were obtained by subtraction of the vdw-, q-. and HB-terms corre- sponding to the refined complex and to the minimized free molecules. Energy minima for compounds 1-4 were determined by a semiempirical method AM1 (as implemented in HyperChem 5.11). The terminal dimethylamine nitrogen of the (dimethylamino)propyl tail ( $\mathrm{p} K_{\mathrm{a}}$ about 9.3 ) of ligands $\mathbf{1 - 4}$ was assumed to be singly protonated. Conformational searching in torsional space was performed using the multiconformer method [19]. Conformations thus obtained were confirmed as minima by vibrational analysis.

The expansion of groove widths has been monitored by measuring $\mathrm{H} 4{ }^{\prime}-\mathrm{H} 5^{\prime}$ interstrand separation [20]. Positions of hydrogen atoms attached to deoxyribose $\mathrm{C}^{\prime}$ ' and $\mathrm{C}^{\prime}$ atoms were generated by HyperChem 5.11, which was also used to calculate interstrand distances between the atoms $\mathrm{H} 4^{\prime}$ and $\mathrm{H}^{\prime}$. Two $\mathrm{H} 4^{\prime}-\mathrm{H} 5^{\prime}$ distances were measured for each nucleotide pair, making a total of 18 distances for each dodecanucleotide duplex. Each $\mathrm{H}^{\prime}{ }^{\prime}$ or $\mathrm{H}^{\prime}$ atom was paired up with a $\mathrm{H} 5^{\prime}$ or $\mathrm{H} 4^{\prime}$ atom on the opposite strand $(n+3)$, nucleosides along the $3^{\prime}$ direction.

\section{RESULTS AND DISCUSSION}

\section{Binding of netropsin, distamycin and its carbocyclic analogues}

The calculated binding energy and component energies for each minimized complex are collected in Table 1. The total binding energy varies between $-75.1 \mathrm{kcal} / \mathrm{mol}$ for distamycin and $-56.3 \mathrm{kcal} / \mathrm{mol}$ for compound 4 . The binding of the compounds 1-2 and 3-4 are predicted to be less favourable by about 10 $\mathrm{kcal} / \mathrm{mol}$ and about $19 \mathrm{kcal} / \mathrm{mol}$, respectively, relative to distamycin. The ranking order for binding (Table 1) is dominated by van der Waals $\left(\Delta E_{\mathrm{vdw}}\right)$ factors, rather than electrostatic $\left(\Delta E_{\mathrm{q}}\right)$ or H-bonded $\left(\Delta E_{\mathrm{HB}}\right)$ terms.

Structural distortions from the standard geometry are expected in the binding of any structurally complex ligand, as the two mole- 
A
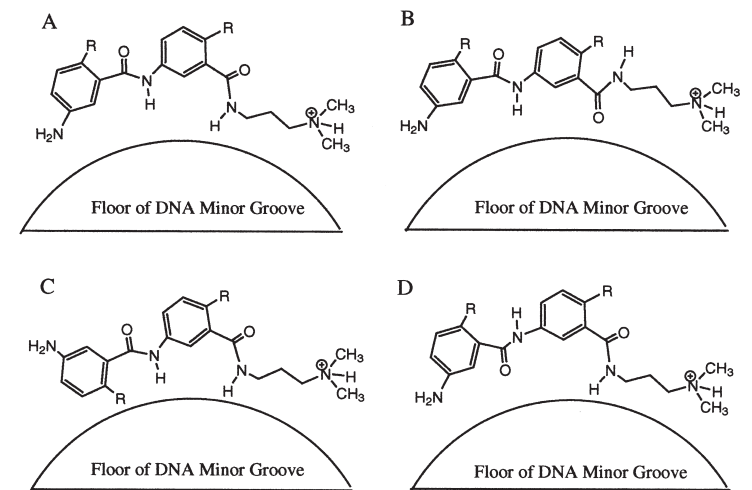

D

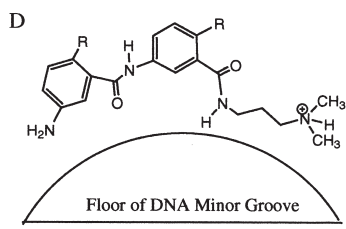

$\mathrm{R}=\mathrm{H}$ or $\mathrm{OCH}_{3}$

B
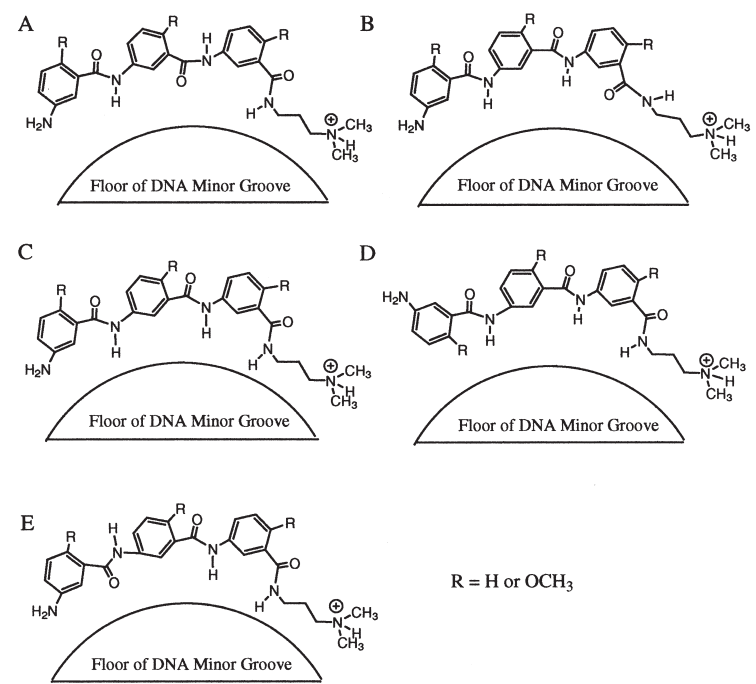

$\mathrm{R}=\mathrm{H}$ or $\mathrm{OCH}_{3}$

Figure 3. Conformation of the studied compounds, showing the distinct orientations available for the amide groups and amine group relative to the DNA.

A, compounds $1(\mathrm{R}=\mathrm{H})$ and $\mathbf{2}\left(\mathrm{R}=\mathrm{OCH}_{3}\right)$; $\mathrm{B}$, compounds $3(\mathrm{R}=\mathrm{H})$ and $4\left(\mathrm{R}=\mathrm{OCH}_{3}\right)$.

cules optimize their structures to maximize the interaction energy. The conformational adaptability of duplex DNA is clearly evident from the wide range of perturbations observed in structures of complexes with proteins and ligands of natural origin [3, 21, 22]. Overall, the d(CGCGAATTCGCG) $)_{2}$ duplex perturbations in minimized complexes are quite modest (Table 1). The ligand induced perturbation is small for netropsin, dista- mycin, 1 and $\mathbf{2}$ models, about $1-3 \mathrm{kcal} / \mathrm{mol}$, with the most extreme effect for compound $\mathbf{4}$, about $5 \mathrm{kcal} / \mathrm{mol}$. It is interesting to note that, for netropsin and distamycin complexes, the hydrogen bond term becomes significant (a contribution of about $-2.7 \mathrm{kcal} / \mathrm{mol}$ ), contrasting with the smaller by about $50 \%$ average values of -1.6 and $-1.2 \mathrm{kcal} / \mathrm{mol}$ for $\mathbf{1 - 2}$ and 3-4, respectively. It should be kept in mind, that the calculated differences in ligand-duplex binding are based on a simple comparison of the binding energy values and do not include important contributions originating from entropic and solvent effects [23-25].

\section{Details of netropsin and distamycin groove-binding interaction with d(CGCGAATTCGCG) 2}

In the structure for the low energy netropsin-d(CGCGAATTCGCG) 2 complex (Fig. 4B) after molecular dynamics refinement the drug molecule is located within the A5-T8 tract of the DNA. In agreement with crystallographic (Fig. 4A), solution NMR, and footprinting studies [26, 27], netropsin occupies a 4-base pair 5'-AATT site. Each amide group of netropsin is positioned approximately midway between two successive base pairs. The amide $\mathrm{NH}$ groups make bifurcated hydrogen bonds to adenine or tymine bases on opposite strands and on two adjacent base pairs, exactly like the water bridges observed in the minor groove spine of hydration in the unliganded DNA [27]. The model for netropsin (Fig. 4B) shows a weak contact, via the N(8) amide group of netropsin with N3 (A18) distance of $3.4 \AA$. An equivalent hydrogen bond is also seen in the crystal structure (Fig. 4A) but is considerably stronger at distance of $2.6 \AA$. This behaviour is due to a small displacement of netropsin relative to the crystal structure position and the more extended conformation adopted by the ligand. In our model, netropsin makes a snug fit to the floor of the minor groove and is bound via a strong 
hydrogen bond. The inner-facing nitrogen of one amidinium group (N9) forms the hydrogen-bond to the N3 A(17) of $3.3 \AA$ length, groove ligands [3]. This result points to an obvious limitation of the present model; as the solvent is not included explicitly in the calcula-

Table 1. Calculated energies for interaction of netropsin, distamycin and compounds 1-4 with d(CGCGAATTCGCG) 2

\begin{tabular}{lcccccc}
\hline Ligand & $\Delta E_{\text {bind }}$ & $\Delta E_{\mathrm{vdw}}$ & $\Delta E_{\mathrm{q}}$ & $\Delta E_{\mathrm{HB}}$ & $\Delta E_{\mathrm{per}}(\mathrm{DNA})$ & $\Delta E_{\text {per }}$ (drug) \\
\cline { 2 - 7 } & \multicolumn{5}{c}{$(\mathrm{kcal} / \mathrm{mol})$} \\
\hline Netropsin & -72.5 & -46.1 & -28.3 & -2.6 & 3.5 & 1.0 \\
Distamycin & -75.1 & -51.9 & -25.2 & -2.9 & 3.8 & 1.1 \\
$\mathbf{1}$ & -64.5 & -45.0 & -24.1 & -1.8 & 4.1 & 2.3 \\
$\mathbf{2}$ & -66.2 & -48.7 & -23.4 & -1.5 & 4.6 & 2.8 \\
$\mathbf{3}$ & -57.7 & -49.7 & -16.8 & -1.3 & 6.0 & 4.1 \\
$\mathbf{4}$ & -56.3 & -49.4 & -17.7 & -1.2 & 6.8 & 5.2 \\
\hline
\end{tabular}

All energies were calculated using the AMBER force field. Refer to Methods for details.

while the inner-facing nitrogen of the other amidinium (N1) is involved in the hydrogen bond to $\mathrm{O} 2$ (C21) of $3.1 \AA$ length, four base pairs away. However, in the crystal structure only one end of netropsin lies deep in the minor groove, and, at the other end of the molecule, the guanidinium group interacts with DNA via a bridging water molecule (PDB file 101D) [27]. This feature has also been observed in the X-ray structures of other minor tions no such binding mode could be predicted. Further, the amidinium and guanidinium groups in our model are twisted out of the planes of the pyrrole rings, by $38^{\circ}$ and $24^{\circ}$, rather than by $0^{\circ}$ and $17^{\circ}$, respectively, found in the crystal structure [26]. However, in view of the $2.2 \AA$ crystallographic resolution and the high temperature factors associated with the bound ligand, such structural differences are unlikely to be significant.
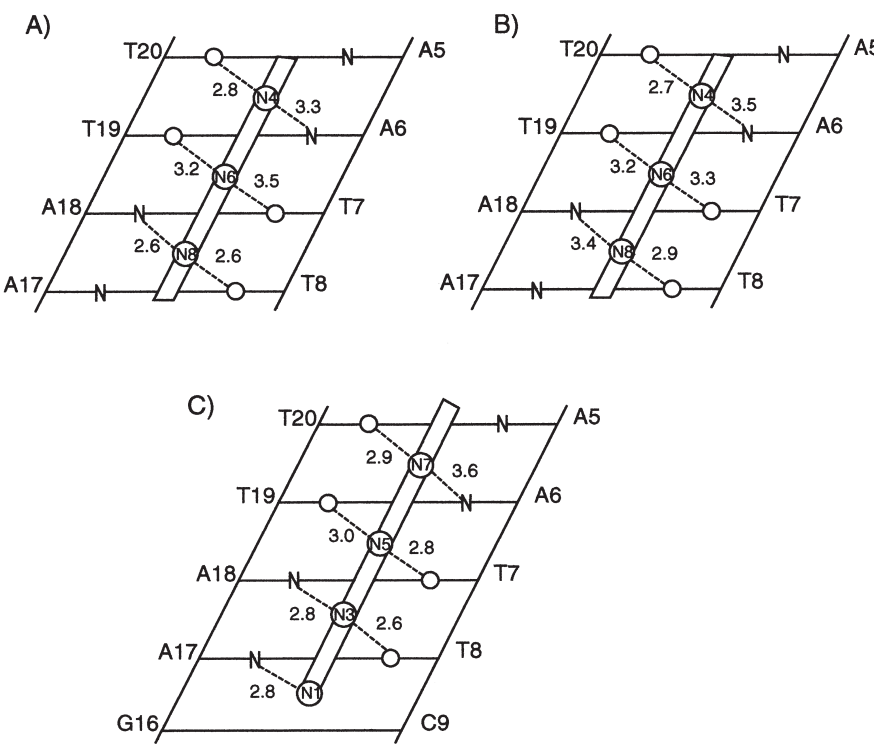

Figure 4. Bifurcated hydrogen bonds between NH of the ligands and DNA minor groove $\mathrm{N}$ and $\mathrm{O}$ atoms.

A, Netropsin-d(CGCGAATT $\left.{ }^{5 \mathrm{Br}} \mathrm{CGCG}\right)_{2}$ crystal structure [22]; B, Low energy netropsin-d(CGCGAATTCGCG) $)_{2}$ complex after MD-refinement; C, Low energy distamycin-d(CGCGAATTCGCG) $)_{2}$ complex after MD-refinement. The distances between potential hydrogen bond donors and acceptors are dashed. Straight segments of the ligands' backbone between numbered amide circles mark the locations of pyrrole. DNA numbering system used was as follows:

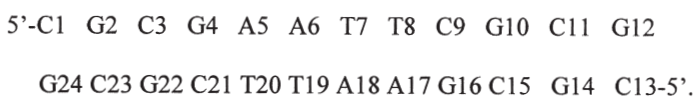

Base pair C1 G24 is at the top of the helix as drawn here. The nitrogen atoms are numbered as in Fig. 1. 
Distamycin forms effectively a centrosymmetric complex (Fig. 4C), with DNA base sequence preferences that resemble those exhibited by netropsin. On account of its larger size, additional hydrogen-bonded contacts, at distances of $3.2 \AA$ and $2.9 \AA$, are made via $\mathrm{N} 1$ to N3 (A17) and N(9) to N3 (A5), respectively. Distamycin makes closer contacts with $\mathrm{O} 2$ (T19) and $\mathrm{O} 2$ (T7) than does netropsin, at distances of 3.0 and $2.8 \AA$, respectively (Fig. $4 \mathrm{C}$ ). These closer contacts are reflected in the van der Waals and hydrogen bond energies determined for distamycin (Table 1). In contrast to the crystal structure of distamycin bound to d(CGCAAATTTGCG) ${ }_{2}$ [27], in our model the entire drug molecule is shifted down the minor groove by approximately one base pair step. This difference in ligand binding to AAATTT and AATT sequences emphasizes the subtle role played by DNA structure in determining the precise location of ligands. Our molecular modelling studies suggest that the formation of hydrogen bonds to guanine N3 atoms is hindered by adenine $\mathrm{NH}_{2}$ group which comes in close contact with pyrrole rings of distamycin.

The calculation of groove width depends on the choice of the reference atom. Consideration of the atoms involved in contacting a ligand suggests that $\mathrm{C}^{\prime}$ ' or $\mathrm{C}^{\prime}$ ' can be more appropriate than the conventional choice of phosphorus atoms [20, 28]. The expansion of groove widths has been monitored by measuring the $\mathrm{H} 4^{\prime}-\mathrm{H} 5^{\prime}$ interstrand distance. The induced widening of the minor groove is evident in the ligand-bound region, compared with the native DNA crystal structure (Fig. 5). Indeed, the induced opening of the A5-C9 minor groove tract is apparent for all the compounds studied, suggesting that increased van der Waals factors may be involved. The minor groove is mostly unchanged in width for representative compound-DNA complexes, as compared with the crystal structure for the netropsin-DNA complex (PDB file 101D), but is opened by about $0.5-1.5 \AA$ in comparison with the native DNA duplex (PDB file 1 bna).
Induced perturbations of the DNA duplex are small and not propagated beyond the immediate binding site.

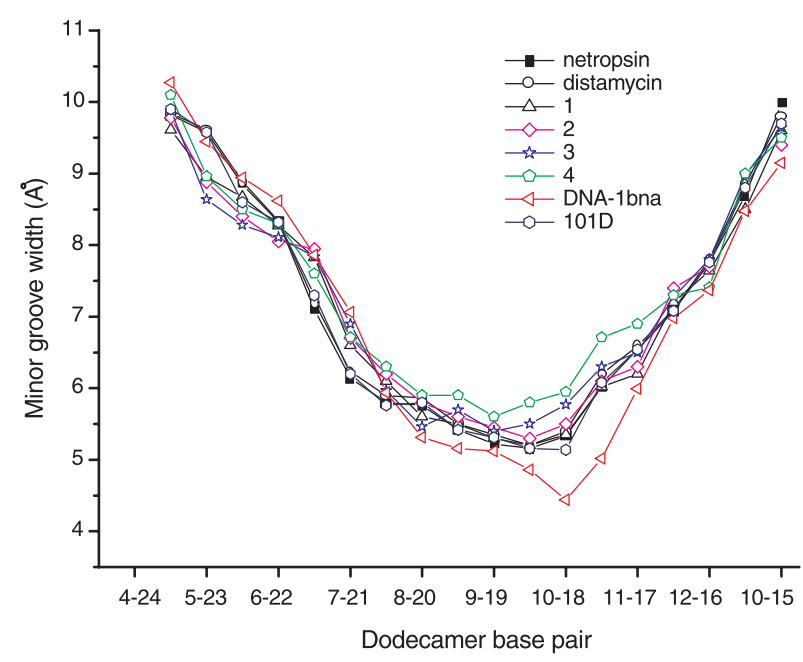

Figure 5. Plots of minor groove width based on H4'-H5' distances (see Methods) for the native d(CGCGAATTCGCG) 2 (DNA-1bna), its crystal complex with netropsin (101D) and complexes of netropsin, distamycin and their carbocyclic analogues 1-4 with d(CGCGAATTCGCG) 2 after MD-refinement.

Dodecamer base pairs are numbered as in Fig. 4.

\section{Details of groove-binding interaction of the carbocyclic analogues of netropsin and distamycin with d(CGCGAATTCGCG) 2}

The structures for the minimized 1-d(CGCGAATTCGCG $)_{2}$ and 2-d(CGCGAATTCG$\mathrm{CG})_{2}$ complex (Fig. 6A and B) shows that the ligands occupy symmetric sites that span 1 fewer base pairs than dose netropsin (in agreement with its shorter length), to give an effective 3-base pair 5'-ATT-3' binding site or recognition sequence. The molecule makes a snug fit to the floor of the minor groove (Fig. 7A and B).

The close fit favors increased van der Waals and H-bonded interactions with the DNA, and inducing a small perturbation of the duplex (Table 1; Fig. 7A and B), which suggests that the molecules $\mathbf{1}$ and $\mathbf{2}$ are of the same isohelicity with the minor groove of the DNA as is netropsin itself. This suggests that short 
homologues of netropsin can be accommodated more deeply into the groove, in agreement with the superior DNA-binding activity observed for 1-2 than for 3-4. The protonated terminal dimethylamine nitrogen of the (dimethylamino)propyl tail is adjacent to a negatively charged phosphodiester linkage. Introduction of a moderately hydrophobic methoxy group at positions ortho to the amide moieties of each phenyl ring of $\mathbf{1}$ would result in close (2.1-2.3 $\AA$ ) contacts between the methoxy $\mathrm{H}$ atoms and sugar $\mathrm{H} 4^{\prime}$, $\mathrm{H} 5^{\prime}$ atoms
Our detailed models reveal that the extent of each binding site for $\mathbf{3}$ and $\mathbf{4}$ is not exactly $4 \mathrm{bp}$, but rather lies somewhere between 3 and $4 \mathrm{bp}$. The benzene rings of 3 and 4 are positioned roughly in the plane of the bases, and amide group are located between base pairs (Fig. 6C and D). Interestingly, neither 3 nor 4 are able to make strong hydrogen-bonded contacts with $\mathrm{A} 6$ and T20 base acceptors located at the floor of the minor groove. When three carboxamide $\mathrm{NH}$ groups of $\mathbf{3}$ and $\mathbf{4}$ face inwards, the strong curvature of $\mathbf{3}$ and $\mathbf{4}$ would
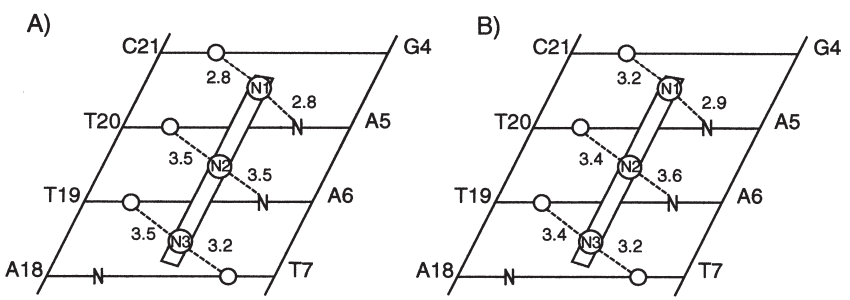

C)

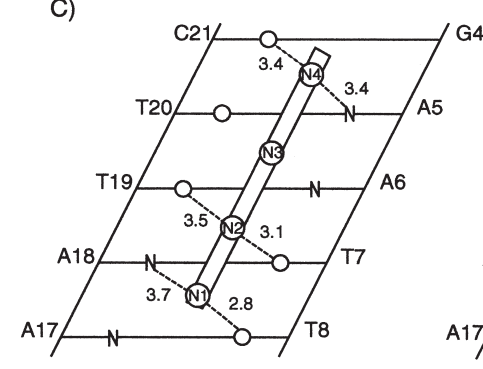

D)
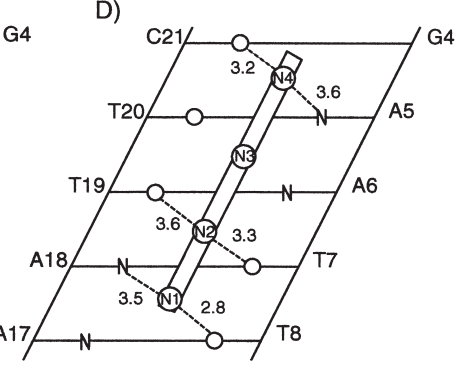

Figure 6. Bifurcated hydrogen bonding between NH on the ligands and DNA minor groove $\mathrm{N}$ and $\mathrm{O}$ atoms.

Low energy complexes between the carbocyclic analogues and d(CGCGAATTCGCG) $)_{2}$, after MD-refinement: A, 1; B, 2; C, 3; D, 4. The distances between potential hydrogen bond donors and acceptors are dashed. Straight segments of the ligands' backbone between numbered amide circles mark the locations of benzenes. The DNA numbering system used was the same as in the legend to Fig. 4. The nitrogen atoms are numbered as in Fig. 2.

of the DNA backbone. These effects are stabilizing the complex. This is reflected in a 2 $\mathrm{kcal} / \mathrm{mol}$ increase in the binding energy of compound $\mathbf{2}$ in comparison to $\mathbf{1}$.

The structures for the minimized $\mathbf{3}$ and 4-d(CGCGAATTCGCG) ${ }_{2}$ complexes (Fig. 6C and D) show that, in the minor groove of DNA, these ligands have an alternative orientation with respect to compounds $\mathbf{1}$ and $\mathbf{2}$. The $\mathbf{3}$ and 4 analogues form centrosymmetric 4-base pair complexes with the ligands displaced towards the $5^{\prime}$ end of the $5^{\prime}$-AATT binding site by approximately one-half a base pair step, relative to netropsin (Fig. 4A and B). This displacement facilitates increased van der Waals contacts with the walls of the minor groove. prevent significant interaction in the minor groove. No regular pattern of bifurcated hydrogen bonds then exists, as shown in Fig. 6C and D. Additional hydrogen-bonded contact of $\mathbf{3}$ and $\mathbf{4}$ in comparison with netropsin, are made at distances of $3.4 \AA$ and $3.2 \AA$, respectively via $\mathrm{NH}_{2}$ (amine) to the $\mathrm{O} 2$ (C21). Our model is consistent with the observed reduced affinity to AT pairs and increased affinity toward GC sequences of the carbocyclic analogues of distamycin and netropsin in comparison with parent compounds [29, 30]. In addition to decreasing affinity for the $5^{\prime}$-AATT-3' match site, there are weaker contacts with the $\mathrm{O} 2$ atom of $\mathrm{C} 21$, indicating that the binding-site size requirement for $\mathbf{3}$ and $\mathbf{4}$ extends 


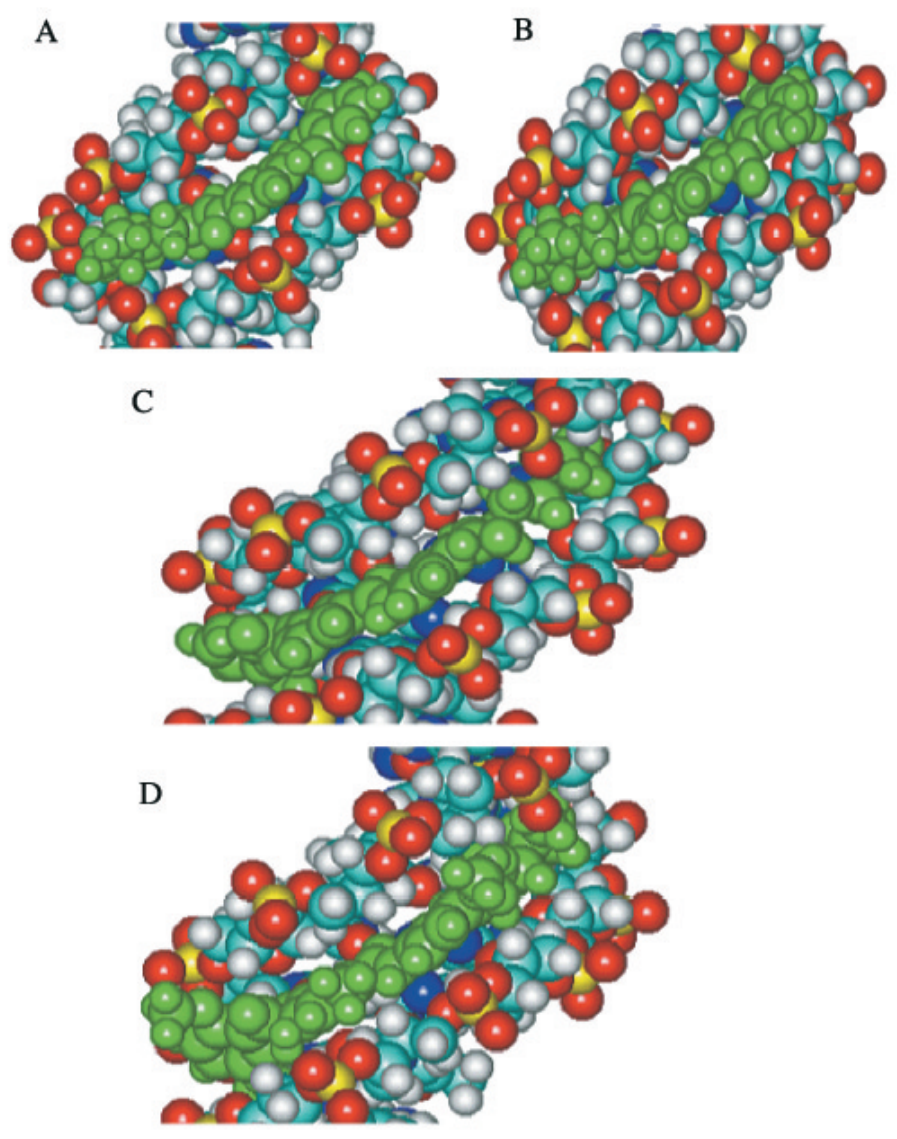

Figure 7. Views of the low energy complexes formed between the d(CGCGAATTCGCG) $)_{2}$ and the carbocyclic analogues of netropsin and distamycin after molecular dynamics refinement.

A, 1; B, 2; C, 3; D, 4. Ligand molecules are shown in green.

over slightly more than the four central AT base pairs. In contrast to the smaller compounds $\mathbf{1}$ and $\mathbf{2}$, the energy wells for these ligands within this AT tract are narrow and the data indicate that specific interactions with flanking sequences strongly inhibit ligand translation along the minor groove. The three benzene rings of $\mathbf{3}$ and $\mathbf{4}$ are slightly twisted so that each ring can be parallel to an appropriate fragment of the enclosing walls of the DNA minor groove (Fig. 7C and D). The compounds 3 and $\mathbf{4}$ produce an increase in groove width of approximately $1.5 \AA$ compared to the netropsin-DNA complex (Fig. 6). The hydrophobic methoxy groups of $\mathbf{4}$ are situated outside the minor groove, therefore the binding energy for $\mathbf{3}$ and $\mathbf{4}$ is almost the same.

An important component of the binding energy that molecular mechanics is unable to fully take into account, involves the role of wa- ter molecules. Neither the angular dependence of the hydrogen bonds nor the hydrogen bond network is modeled in the classical force field. It is possible that the model could be improved by the inclusion of explicit solvent in the molecular modelling calculations because, in the crystal structure, water molecules are located in the minor groove vicinity of the bound netropsin [26].

An accurate definition by molecular modelling of the optimal binding site for the compounds studied alone has been hampered by the fact that the DNA fragment used in the model contains a limited number of binding sites. Various dodecamer sequences, even though they exhibit a similar general trend, show much dissimilarity in the detail of their structure [3, 21, 31, 32]. Analysis of DNA complexes with netropsin, distamycin, and their analogues led to debate over the contributions 
of H-bonding, electrostatic, and van der Waals interactions to minor groove binding affinity and specificity. To design improved drugs that target the minor groove of DNA, it is essential to have a more detailed understanding of such interactions. It is obvious that the interaction model presented here should be validated by accurate physical measurements. The availability of additional experimentally determined data sets should help to further validate the model and simulations.

\section{CONCLUSIONS}

Our molecular modelling approach, using the d(CGCGAATTCGCG) ${ }_{2}$ duplex as a model DNA host, provides a qualitative method for comparing the binding properties of carbocyclic analogues of netropsin and distamycin. For netropsin itself, this procedure suggests a drug conformation that is closely related to that in the crystal structure of the complex [27]. We predict that analogues of netropsin $\mathbf{1}$ and $\mathbf{2}$ would be effectively isohelical with the DNA minor groove. The superior DNA-binding afforded by compounds $\mathbf{1}$ and $\mathbf{2}$ with respect to $\mathbf{3}$ and $\mathbf{4}$ results from their more effective penetration into the minor groove and reduced perturbation factors. From the analysis of our model it appears that van der Waals and electrostatic interactions are more important in stabilizing the complexes than specific hydrogen bonds formation.

\section{R E F E R E N C E S}

1. Nielsen, E.P. (1991) Sequence-selective DNA recognition by synthetic ligands. Bioconjugate Chem. 2, 1-12.

2. Bailly, Ch. \& Chaires, J.B. (1998) Sequencespecific DNA minor groove binders. Design and synthesis of netropsin and distamycin analogues. Bioconjugate Chem. 9, 513-538.
3. Neidle, S. (1997) Crystallographic insights into DNA minor groove recognition by drugs. Biopolymers 44, 105-121.

4. Tanious, F.A., Spychala, J., Kumar, A., Greene, K., Boykin, D.W. \& Wilson, W.D. (1994) Different binding mode in AT and GC sequences for unfused-aromatic dications. $J$. Biomol. Struct. Dyn. 11, 1063-1083.

5. Kopka, M.L., Yoon, C., Goodsell, D., Pjura, P. \& Dickerson, R.E. (1985) The molecular origin of DNA-drug specificity of netropsin and distamycin. Proc. Natl. Acad. Sci. U.S.A. 82, 1376-1380.

6. Zakrzewska, K., Lavery, R. \& Pullman, B. (1984) Theoretical studies of the selective binding to DNA of two nonintercalating ligands: Netropsin and SN 18071. Nucleic Acids Res. 11, 8825-8839.

7. Lown, J.W. (1995) Design and development of sequence selective lexitropsin DNA minor groove binders. Drug Development Res. 34, 145-183.

8. Walker, W.L., Kopka, M.L. \& Goodsell, D.S. (1997) Progress in the design of DNA sequence-specific lexitropsins. Biopolymers 44, 323-334.

9. Swalley, S.E., Baird, E.E. \& Dervan, P.B. (1999) Effects of $\gamma$-turn and $\beta$-tail amino acids on sequence-specific recognition of DNA by hairpin polyamides. J. Am. Chem. Soc. 121, 1113-1129.

10. Bartulewicz, D., Bielawski, K., Markowska, A., Zwierz, K. \& Różański, A. (1998) Synthetic analogues of netropsin and distamycin - synthesis of a new pyridine and carbocyclic analogues of the pyrrolecarboxamide antitumour antibiotics. Acta Biochim. Polon. 45, 41-57.

11. Turner, P.R., Ferguson, L.R. \& Denny, W.A. (1999) Polybenzamide mustards; Structureactivity relationships for DNA sequence-specific alkylation. Anti-Cancer Drug Des. 14, 61-70. 
12. Bartulewicz, D., Wołczyński, S., Dabrowska, M. \& Różański, A. (2000) Molecular modelling, synthesis and antitumor activity of carbocyclic analogues of netropsin and distamycin - new carriers of alkylating elements. Acta Biochim. Polon. 47, 23-35.

13. Dickerson, R.E. \& Drew, H.R. (1981) Structure of a B-DNA dodecamer II. Influence of base sequence on helix structure. J. Mol. Biol. 149, 761-786.

14. Flatters, D., Zakrzewska, K. \& Lavery, R. (1997) Internal coordinate modeling of DNA: Force field comparisons. J. Comput. Chem. 18, 1043-1055.

15. Weiner, S.J., Kollman, P.A., Case, D.A., Singh, U.C., Ghio, G., Alagano, G., Profeta, S. \& Weiner, P. (1984) A new force-field for molecular mechanical simulation of nucleic acids and proteins. J. Am. Chem. Soc. 108, 765784.

16. Weiner, S.J., Kollman, P.A., Nguyen, D.T. \& Case, D.A. (1986) An all atom force field for simulations of proteins and nucleic acids. $J$. Comput. Chem. 7, 230-252.

17. Orozco, M. \& Luque, F.J. (1990) On the use of AM1 and MNDO wavefunctions to compute accurate electrostatic charges. J. Comput. Chem. 11, 909-923.

18. Orozco, M., Laughton, C.A., Herzyk, P. \& Neidle, S. (1990) Molecular modelling of drug-DNA structures: The effects of differing dielectric treatment on helix parameters and comparison with a fully solvated structural model. J. Biomol. Struct. Dyn. 8, 359-373.

19. Lipton, M. \& Still, W.C. (1988) The multiple minimum problem in molecular modeling. Tree searching internal coordinate conformational space. J. Comput. Chem. 9, 345-355.

20. Neidle, S. (1992) Minor-groove width and accessibility in B-DNA drug and protein complexes. FEBS Lett. 298, 97-99.
21. Berman, H.M. (1997) Crystal studies of B-DNA: The answers and the questions. Biopolymers 44, 23-44.

22. Laughton, Ch. \& Luisi, B. (1999) The mechanics of minor groove width variation in DNA, and its implications for the accommodation of ligands. J. Mol. Biol. 288, 953-963.

23. Marky, L. \& Breslauer, K.J. (1987) Origins of netropsin binding affinity and specificity: Correlations of the thermodynamic and structural data. Proc. Natl. Acad. Sci. U.S.A. 84, 43594363.

24. Young, M.A., Jayaram, B. \& Beveridge, D.L. (1997) Intrusion of counterions into the spine of hydration in the minor groove of B-DNA: Fractional occupancy of electronegative pockets. J. Am. Chem. Soc. 119, 59-69.

25. Singh, S.B. \& Kollman, P.A. (1999) Calculating the absolute free energy of association of netropsin and DNA. J. Am. Chem. Soc. 121, 3267-3271.

26. Goodsell, D.S., Kopka, M.L. \& Dickerson, R.E. (1995) Refinement of netropsin bound to DNA: Bias and feedback in electron density map interpretation. Biochemistry 34, 49834993.

27. Kopka, M.L., Yoon, C., Goodsell, D., Pjura, P. \& Dickerson, R.E. (1985) The molecular origin of DNA-drug specificity in netropsin and distamycin. Proc. Natl. Acad. Sci. U.S.A. 82, 1376-1380.

28. Mazur, A.K. (1999) Internal correlations in minor groove profiles of experimental and computed B-DNA conformations. J. Mol. Biol. 290, 373-377.

29. Dasgupta, D., Rajagopalan, M. \& Sasisekharan, V. (1986) DNA-binding characteristics of a synthetic analogue of distamycin. Biochim. Biophys. Res. Commun. 140, 626-631.

30. Yan, Y., Liu, M. \& Gong, B. (1997) Two-ring DNA minor-groove binders consisting of 
readily available, di-substituted benzene derivatives. Bioorg. Med. Chem. Lett. 7, 1469-1474.

31. Johannesson, H. \& Halle, B. (1998) Minor groove hydration of DNA in solution: Dependence on base composition and sequence. $J$. Am. Chem. Soc. 120, 6859-6870.
32. Tereshko, V., Minasov, G. \& Egli, M. (1999) A "hydration" spine in a B-DNA minor groove. $J$. Am. Chem. Soc. 121, 3590-3595. 